\title{
Acompanhamento farmacoterapêutico dos pacientes com leucemia mieloide crônica em uso de mesilato de imatinibe na Universidade Federal do Ceará
}

\author{
The pharmacotherapeutic follow-up of patients with chronic myeloid leukemia (CML) on imatinib \\ mesylate therapy
}

Sterfen S. Aquino ${ }^{1}$

Romélia P. Gonçalves ${ }^{2}$

Lilianne B. Silva ${ }^{3}$
Leucemia mieloide crônica (LMC) é uma desordem genética de etiologia desconhecida, caracterizada por crescimento aumentado e não regulado de células precursoras mieloides na medula óssea. LMC está associada com uma característica translocação cromossômica chamada de cromossoma Philadelphia. Esse é um estudo observacional descritivo de pacientes com LMC do Hospital Universitário Walter Cantídio, Universidade Federal de Ceará, Brasil. O objetivo foi estudar a eficácia e a frequência de efeitos colaterais da terapia com mesilato de imatinibe. Vinte e seis pacientes foram incluidos: 09 em fase crônica (34,61\%), 06 em fase acelerada (23,08\%) e 11 em crise blástica $(42,31 \%)$. Os casos em fase crônica tiveram intolerância prévia para interferon alfa (IFN- $\alpha$ ). Resposta hematológica completa foi observada em sete pacientes, cinco em fase crônica, um em acelerada e um em crise blástica. Durante o primeiro ano de tratamento, quatro pacientes em fase crônica mostraram resposta citogenética completa. Um destes pacientes perdeu a resposta posteriormente. Nenhum paciente em fase acelerada ou crise blástica mostrou resposta citogenética completa. Entre os 18 pacientes que estavam vivos no fim do estudo, apenas quatro (22,22\%) não tiveram nenhuma queixa. Os mais comuns efeitos adversos foram: edema (50\%), adinamia (33,33\%), dor óssea elou articular (33,33\%), cefaléia (27,78\%), cãimbra (16,67\%), diarreia (16,67\%), insônia $(16,67 \%)$, prurido $(16,67 \%)$, equimoses $(11,11 \%)$, náuseas $(11,11 \%)$, dor epigástrica $(5,55 \%)$, eritema $(5,55 \%)$, lacrimejamento $(5,55 \%)$, ressecamento da pele e lábios (5,55\%), rush (5,55\%), sudorese (5,55\%). Uma minoria de pacientes desenvolveu resistência ao imatinibe. Para superar a resistência e aumentar a resposta positiva aos inibidores de tirosino-quinase novos fármacos e testes estão sendo utilizados e pesquisados. Rev. Bras. Hematol. Hemoter. 2009; 31(3):137-142

Key words: Leucemia mieloide crônica; mesilato de imatinibe; acompanhamento farmacoterapêtico.

\section{Introdução}

A leucemia mieloide crônica (LMC) é uma doença de etiologia desconhecida, caracterizada por alteração cromossômica bem estudada. É caracterizada pela presença da translocação entre os braços longos dos cromossomos
9 e 22 (cromossomo Philadelphia). O gene resultante BCRABL apresenta atividade tirosina-quinase e é responsável pelo processo oncogênico, desencadeando o início de várias cascatas de sinalização envolvidas no crescimento, na diferenciação, na adesão e na morte celular. ${ }^{1,2} \mathrm{O}$ mesilato de imatinibe é um inibidor potente e específico de todas as

\footnotetext{
Universidade Federal do Ceará - Fortaleza-CE.

Correspondência: Lilianne Brito da Silva

Rua Doutor José Lourenço 1820, apto. 502, Aldeota.

60.115-281 - Fortaleza-CE - Brasil

E-mail: liliannebrito@hotmail.com

Doi: 10.1590/S1516-84842009005000048
}

${ }^{1}$ Aluno da Graduação de Farmácia da Universidade Federal do Ceará - Fortaleza-CE.

${ }^{2}$ Professora Adjunta da Universidade Federal do Ceará - Fortaleza-CE.

${ }^{3}$ Médica Hematologista e Hemoterapeuta. Aluna de Pós-graduação em Patologia Tropical da Universidade Federal do Ceará - Fortaleza-CE. 
quinases relacionadas ao $\mathrm{ABL}$, competindo pelos sítios de ligação da enzima BCR-ABL tirosina-quinase com o ATP, o qual leva à inibição da fosforilação da tirosina das proteínas envolvidas na transdução do sinal BCR-ABL. ${ }^{3}$ Esta substância mostra um alto grau de especificidade para a BCR$\mathrm{ABL}$; para o receptor do fator de crescimento plaquetário e o receptor C-Kit tirosina-quinase, que promove a proliferação de células de tumores do estroma gastrointestinal (GIST), causando parada do crescimento e apoptose nas células hematopoéticas que expressam o BCR-ABL, mas não afetam as células normais. ${ }^{3,4,5} \mathrm{O}$ mesilato de imatinibe é indicado para o tratamento de pacientes com LMC em crise blástica, fase acelerada, ou em fase crônica em caso de resistência ou intolerância ao uso de interferon-alfa. Também está indicado para o tratamento de tumores estromais gastrointestinais malignos irressecáveis e/ou metastáticos. ${ }^{6,7}$

O presente estudo teve como objetivo analisar a ação e os efeitos colaterais do mesilato de imatinibe nos pacientes portadores de LMC provenientes de um serviço especializado.

\section{Casuística e Método}

Trata-se de estudo observacional descritivo em pacientes com LMC em tratamento com mesilato de imatinibe, de abordagem quantitativa. Foram incluídos no estudo 26 pacientes portadores de LMC, sendo nove em fase crônica $(34,61 \%)$, seis em fase acelerada $(23,08 \%)$ e $11 \mathrm{em}$ crise blástica (42,31\%). Conforme os critérios estabelecidos no Protocolo de Diretrizes Terapêuticas (Portaria SAS/MS n ${ }^{\circ}$ 431, de 03/10/2001), os pacientes portadores de LMC podem se beneficiar do tratamento com mesilato de imatinibe quando se apresentarem na fase crônica da patologia após evidência de resistência ou intolerância ao interferon-alfa ou se apresentarem nas fases avançadas, ou seja, fase acelerada ou crise blástica. Na fase crônica, a dose inicial é de $400 \mathrm{mg} \mathrm{VO/dia;} \mathrm{nas} \mathrm{fases} \mathrm{avançadas,}$ a dose inicial prevista é maior, totalizando $600 \mathrm{mg} \mathrm{VO} /$ dia. Tais doses foram modificadas ao longo do estudo de acordo com a necessidade de cada situação. Em nenhum momento foi utilizada dose abaixo de $300 \mathrm{mg} / \mathrm{dia}$. Na ocorrência de mielotoxicidade com a dose mínima de $300 \mathrm{mg} /$ dia, a medicação foi suspensa, com avaliação posterior, objetivando a reintrodução do medicamento logo que possível. ${ }^{8}$ Considerou-se como fonte de dados o prontuário.

Foi elaborado Termo de Consentimento Livre e Esclarecido para os pacientes, atendendo ao que determina a Resolução $n^{\circ}$ 196/96 do Conselho Nacional de Saúde. Foram estudados todos os pacientes portadores de LMC, do serviço mencionado, que estavam no protocolo, com o mesilato de imatinibe no período de janeiro de 2005 , sendo estes seguidos até fevereiro de 2006.

\section{Análise estatística}

Os dados foram plotados na planilha Excel 2000 e analisados no programa SPSS (Statistical Package for the Social Sciences) versão 10.0.

\section{Resultados}

A mediana da idade dos pacientes da amostra foi de 50 anos (26-77), 53,85\% do sexo masculino. A mediana do tempo transcorrido entre o diagnóstico da doença e a inclusão no protocolo com o mesilato de imatinibe foi de 45 meses (6-216) para fase crônica, 38 meses (5-71) para a fase acelerada e 20 meses (1-69) para a crise blástica. No início do protocolo, foram observadas esplenomegalia em cinco pacientes $(50 \%)$ e hepatoesplenomegalia em cinco pacientes (50\%) (Tabela 1).

Resposta hematológica completa foi observada em sete pacientes, sendo cinco na fase crônica, um na acelerada e um na crise blástica. Durante o primeiro ano de tratamento com o mesilato de imatinibe, quatro pacientes da fase crônica apresentaram resposta citogenética completa, ocorrendo perda de resposta completa adquirida em um paciente. $\mathrm{Na}$ fase acelerada e crise blástica, nenhum dos pacientes apresentou resposta citogenética completa (Tabela 2). A avaliação da resposta molecular não está disponível no serviço como ferramenta de acompanhamento da resposta ao uso da medicação.

Dentre os cinco pacientes em fase crônica que estavam em resposta hematológica completa, dois possuíam níveis de hemoglobina abaixo de $13 \mathrm{~g} / \mathrm{dL}$ (menor valor registrado: $12,4 \mathrm{~g} / \mathrm{dL}$ ) e um possuía a contagem de plaquetas abaixo de

Tabela 1. Perfil dos pacientes em uso de mesilato de imatinibe

\begin{tabular}{|c|c|c|c|c|}
\hline & $\begin{array}{l}\text { Fase } \\
\text { crônica }\end{array}$ & $\begin{array}{c}\text { Fase } \\
\text { acelerada }\end{array}$ & $\begin{array}{c}\text { Crise } \\
\text { blástica }\end{array}$ & Total $(n=26)$ \\
\hline Mediana da idade (anos) & 52 & 44 & 48 & $50(26-77)$ \\
\hline $\begin{array}{l}\text { Sexo } \\
\text { Masculino } \\
\text { Feminino }\end{array}$ & $\begin{array}{l}6 \\
3\end{array}$ & $\begin{array}{l}3 \\
3\end{array}$ & $\begin{array}{l}5 \\
6\end{array}$ & $\begin{array}{l}14(53,85 \%) \\
12(46,15 \%)\end{array}$ \\
\hline $\begin{array}{l}\text { Mediana do tempo transcorrido } \\
\text { entre o diagnóstico e inclusão } \\
\text { no protocolo com o } \\
\text { mesilato de imatinibe (meses) }\end{array}$ & 45 & 38 & 20 & $35(1-216)$ \\
\hline \multicolumn{5}{|l|}{ Órgãos palpáveis } \\
\hline Fígado & 0 & 0 & 0 & $0(0,0 \%)$ \\
\hline Baço & 2 & 2 & 1 & $5(50 \%)$ \\
\hline Fígado e Baço & 0 & 1 & 4 & $5(50 \%)$ \\
\hline
\end{tabular}

Fonte: Serviço especializado, 2006 
$150 \times 10^{3} / \mathrm{mm}^{3}$. Havia três pacientes com resposta citogenética completa.

O paciente em fase acelerada com resposta hematológica completa possuía nível de hemoglobina abaixo de $13 \mathrm{~g} / \mathrm{dL}$ (valor registrado: $11,6 \mathrm{~g} / \mathrm{dL}$ ); com o nível de plaqueta acima de $150 \times 10^{3} / \mathrm{mm}^{3}$ e abaixo de $400 \times 10^{3} / \mathrm{mm}^{3}\left(336 \times 10^{3} / \mathrm{mm}^{3}\right)$.

O paciente em crise blástica com resposta hematológica completa possuía nível de hemoglobina abaixo de $13 \mathrm{~g} / \mathrm{dL}$ (valor registrado: 10,6 $\mathrm{g} / \mathrm{dL}$ ); com contagem de plaquetas acima de $400 \times 10^{3} / \mathrm{mm}^{3}\left(528 \times 10^{3} / \mathrm{mm}^{3}\right)$.

Anomalias cromossômicas adicionais à translocação t $(9 ; 22)$ foram observadas em três pacientes sendo um em

Tabela 2. Resposta hematológica e citogenética completa dos pacientes com LMC em uso de mesilato de imatinibe

\begin{tabular}{lcccc}
\hline & $\begin{array}{c}\text { Fase } \\
\text { crônica } \\
(n=9)\end{array}$ & $\begin{array}{c}\text { Fase } \\
\text { acelerada } \\
(n=6)\end{array}$ & $\begin{array}{c}\text { Crise } \\
\text { blástica } \\
(n=11)\end{array}$ & $\begin{array}{c}\text { Total } \\
(\mathrm{n}=26)\end{array}$ \\
\hline $\begin{array}{l}\text { Resposta } \\
\text { hematológica completa }\end{array}$ & $\begin{array}{c}5 / 9 \\
(78 \%)\end{array}$ & $\begin{array}{c}1 / 6 \\
(17 \%)\end{array}$ & $\begin{array}{c}1 / 11 \\
(9 \%)\end{array}$ & $\begin{array}{c}7 / 26 \\
(27 \%)\end{array}$ \\
$\begin{array}{l}\text { Resposta citogenética } \\
\text { completa }\end{array}$ & $\begin{array}{c}4 / 9 \\
(44 \%)\end{array}$ & 0 & 0 & $\begin{array}{c}4 / 26 \\
(15 \%)\end{array}$ \\
\hline
\end{tabular}

Fonte: Serviço especializado, 2006

Tabela 3. Anomalias cromossômicas

\begin{tabular}{lcccc}
\hline \multicolumn{1}{c}{ Características } & $\begin{array}{c}\text { Fase } \\
\text { crônica }\end{array}$ & $\begin{array}{c}\text { Fase } \\
\text { acelerada }\end{array}$ & $\begin{array}{c}\text { Crise } \\
\text { blástica }\end{array}$ & $\begin{array}{c}\text { Total } \\
(\mathrm{n}=26)\end{array}$ \\
\hline $\begin{array}{l}\text { Anormalidades } \\
\text { cromossômicas }\end{array}$ & 1 & 0 & 2 & 3 \\
$\begin{array}{l}\text { Evolução clonal com o } \\
\text { Glivec® }\end{array}$ & 0 & 0 & 1 & 1 \\
$\begin{array}{l}\text { Deleção do } 9 \\
\text { Duplo Ph' }\end{array}$ & 1 & 0 & 0 & 1 \\
\hline
\end{tabular}

Fonte: Serviço especializado, 2006

Tabela 4. Total de óbitos em 24 meses

\begin{tabular}{|c|c|c|}
\hline \multirow{2}{*}{$\begin{array}{l}\text { Período } \\
\text { (meses) }\end{array}$} & \multicolumn{2}{|c|}{ Crise blástica } \\
\hline & $\mathrm{N}^{\circ}$ & $\%$ \\
\hline 0 a 6 & 6 & 75 \\
\hline 7 a 12 & 1 & 12,5 \\
\hline 13 a 24 & 1 & 12,5 \\
\hline Total & 8 & 100 \\
\hline
\end{tabular}

Fonte: Serviço especializado, 2006 fase crônica e dois em crise blástica. Apenas em um paciente (em crise blástica) estas alterações foram adquiridas durante o tratamento com o mesilato de imatinibe. As anomalias apresentadas foram: deleção do cromossomo 9 (um paciente em fase crônica, antes do tratamento) e duplo cromossomo $\mathrm{Ph}$ (dois pacientes em crise blástica, sendo um paciente antes do tratamento e um após tratamento com o mesilato de imatinibe). Tabela 3

Todos os óbitos observados durante a pesquisa foram de pacientes em crise blástica. Nos primeiros seis meses de tratamento observaram-se seis óbitos; entre 7 a 12 meses, um óbito; e entre 13 a 24 meses, um óbito (Tabela 4). Foi observada uma média de quatro meses de duração da resposta hematológica completa após o início do uso de mesilato de imatinibe nos pacientes que foram a óbito; após este período do tratamento, estes pacientes voltaram a exibir perfil hematológico de doença em crise blástica.

Dentre os 18 pacientes vivos, apenas quatro $(22,22 \%)$ não relataram reações adversas ao mesilato de imatinibe. As queixas mais recorrentes foram: nove apresentaram edema (50\%); seis, adinamia (33,33\%); seis, dor óssea e/ou articular (33,33\%); cinco, cefaléia (27,78\%); três, cãimbra (16,67\%); três, diarreia (16,67\%); três, insônia $(16,67 \%)$; três, prurido $(16,67 \%)$; dois, equimose $(11,11 \%)$; dois, náusea $(11,11 \%)$; um, epigastralgia $(5,55 \%)$; um, eritema $(5,55 \%)$; um, lacrimejamento $(5,55 \%)$; um, ressecamento da pele e lábios $(5,55 \%)$; um, rush (5,55\%); um, sudorese (5,55\%).

Durante a evolução, as doses da medicação foram modificadas; os pacientes em fase crônica em geral fizeram uso contínuo da dose de $400 \mathrm{mg}$ (um paciente utilizou $300 \mathrm{mg}$ e um utilizou $600 \mathrm{mg}$ ). Dentre os pacientes em fase acelerada, a maioria utilizou a dose de $600 \mathrm{mg}$ (um paciente utilizou $300 \mathrm{mg}$ e um $400 \mathrm{mg}$ ). Em crise blástica, um paciente utilizou $400 \mathrm{mg}$, um $600 \mathrm{mg}$ e um $800 \mathrm{mg}$. Na fase acelerada e na crise blástica houve a necessidade de mudar com maior frequência as dosagens.

A interrupção temporária da medicação ocorreu em várias ocasiões por motivo de mielotoxicidade e citopenias.

\section{Discussão}

A LMC é mais comum após os 50 anos, é bastante indolente, em geral manifestando-se com um acentuado aumento dos leucócitos e às vezes de plaquetas. Geralmente o paciente tem poucos sintomas; um deles pode ser desconforto abdominal, por aumento do baço. O tratamento inclui quimioterapia oral, alfa-interferon, e atualmente o mesilato de imatinibe. O transplante de medula óssea é uma alternativa terapêutica que pode ser curativa, apesar de exibir uma alta mortalidade precoce relacionada ao tratamento. ${ }^{8}$ 
A evolução da LMC ocorre em três fases: a fase crônica, definida por um aumento na contagem dos leucócitos $\geq 20 \times 10^{3} \mu \mathrm{L}$ e menos de $10 \%$ de blastos em sangue periférico ou medula óssea, os sinais e sintomas no início são leves tornando-se piores com a progressão da doença, dura aproximadamente cinco a seis anos; a fase acelerada, definida pela presença de $10 \%$ a $30 \%$ de células blásticas no sangue periférico ou medula óssea, anemia e trombocitopenia, os sintomas geralmente são de média intensidade e incluem febre de origem desconhecida, dor óssea, náuseas e dor abdominal devido à esplenomegalia e ou hepatomegalia, a citogenética pode apresentar nova ou múltiplas anormalidades cromossômicas, dura aproximadamente de seis a nove meses; e a fase blástica, ou crise blástica, definida pela presença de mais de $30 \%$ de blastos em sangue periférico ou medula óssea, estando, dentre os sintomas, a fadiga relacionada à anemia, sangramento, infecção, linfadenopatia e disfunção do sistema nervoso central; esta fase é rapidamente fatal, com uma média de sobrevida de três a seis meses. ${ }^{8}$

Embora a maioria dos pacientes manifeste a doença na fase crônica e avance para a fase acelerada, aproximadamente $25 \%$ dos pacientes progridem diretamente da fase crônica para a fase blástica sem evidência de transição pela fase acelerada $^{8}$

Em relação ao tratamento da LMC existem critérios de resposta importantes que devem ser definidos. Resposta hematológica completa ocorre quando o paciente apresenta após o tratamento: contagem de plaquetas $<450.000 / \mathrm{mm}^{3}$, contagem de leucócitos $<10.000 / \mathrm{mm}^{3}$, ausência de granulócitos imaturos, basófilos $<5 \%$ e baço não palpável. A resposta citogenética é completa quando o paciente não possui células $\mathrm{Ph}$ ` positivas, parcial quando o paciente possui de $1 \%$ a $35 \%$ de células $\mathrm{Ph}$ ' positivas, menor quando há presença de $36 \%$ a $65 \%$ de células $\mathrm{Ph}$ positivas, mínima quando há presença de $66 \%$ a $95 \%$ de células $\mathrm{Ph}$ ' positivas e ausente quando há presença de $>95 \%$ de células $\mathrm{Ph}$ positivas. A resposta molecular é completa quando o paciente possui BCR-ABL não detectável e não quantificável e maior quando o paciente possui a relação BCR-ABL/gene controle com ratio menor ou igual a $0,10 .^{9}$

Para definir a eficácia do protocolo escolhido é importante relacionar o tempo com a resposta do tratamento para LMC. Um tratamento é considerado falho quando: após três meses não há resposta hematológica; após seis meses obtémse menos do que a resposta hematológica completa; após 12 meses há a presença de mais de 35\% de células $\mathrm{Ph}$ ` positivas e, após 18 meses, obtém-se menos do que a resposta citogenética completa. Um tratamento é considerado subótimo quando após três meses obtém-se menos do que a resposta hematológica completa, após seis meses há a presença de mais de 35\% de células Ph positivas, após 12 meses obtémse menos do que a resposta citogenética completa e após 18 meses obtém-se menos do que a resposta molecular maior. ${ }^{9}$
Na década de 80, a droga de eleição era o interferonalfa (IFN- $\alpha$ ), uma citocina natural usada na forma pura (IFN- $\alpha$ ) ou na forma recombinada IFN-2 $\alpha$ (roferon) de administração subcutânea, cujo mecanismo hipotético de ação na LMC é o controle da proliferação, da adesão e da apoptose das células $\mathrm{Ph}^{\prime}$ positivas. ${ }^{10}$

Os primeiros relatos da literatura sobre o uso do imatinibe enfocaram a discussão da superioridade da resposta citogenética e hematológica em relação ao tratamento anterior com interferon-alfa e citarabina associados, apresentando também uma menor toxicidade. ${ }^{11}$

A mediana da idade dos pacientes da amostra foi de 50 anos, sendo 53,85\% do sexo masculino. A mediana do tempo transcorrido entre o diagnóstico da doença e a inclusão no protocolo com o mesilato de imatinibe foi de 45 meses para fase crônica, 38 meses para a fase acelerada e 20 meses para a crise blástica. Este resultado é semelhante a um trabalho em que a idade média dos pacientes foi de 48,41 anos, sendo 60,6\% do sexo masculino. Em relação ao mesmo trabalho, houve diferenças entre o tempo transcorrido entre o diagnóstico da doença e a inclusão no protocolo, pois foi em média de 35 meses para a fase crônica, 62 meses (2-150) para a fase acelerada e 35 meses (3-109) para a crise blástica. ${ }^{12}$

Nos pacientes que foram a óbito, o fator de maior interferência no prognóstico foi o tipo de resposta ao tratamento. Foi observada uma média de quatro meses de duração da resposta hematológica completa após o início do uso de mesilato de imatinibe nos pacientes que foram a óbito; após este período, estes pacientes voltaram a exibir perfil hematológico de doença em crise blástica. Estes achados são semelhantes ao trabalho de O'Dwyer. ${ }^{13}$

$\mathrm{O}$ número de óbitos tem uma maior incidência nos pacientes em crise blástica, pois nesta fase observa-se um maior número de mecanismos de resistência à medicação, que muitas vezes são intransponíveis.

Os pacientes em fase crônica apresentaram uma resposta hematológica menor $(55,55 \%)$ ao encontrado na literatura. Tsao et al. (2002) mostraram anteriormente que, num grupo de 54 pacientes em fase crônica, 98\% $(n=53)$ tiveram resposta hematológica tomando $300 \mathrm{mg} / \mathrm{dia}$ ou mais de medicamento. Deve-se ressaltar também que a resposta citogenética encontrada em nosso estudo foi superior $(44,44 \%)$ à encontrada na literatura. Tsao et al. mostraram que, entre os 54 pacientes em fase crônica, 31\% $(n=17)$ tiveram uma resposta citogenética completa. ${ }^{14}$

Os pacientes em fase acelerada apresentaram uma resposta hematológica inferior $(16,67 \%)$ à descrita na literatura anterior: Tsao et al. mostraram que, dos 181 pacientes incluídos na pesquisa, 34\% tiveram resposta hematológica tomando $600 \mathrm{mg} /$ dia do medicamento. ${ }^{14}$

Deve-se lembrar que o tempo de seguimento nos estudos anteriores é superior ao nosso trabalho, o que poderia influenciar nos resultados obtidos. 
Analisando-se o intervalo entre o diagnóstico e o tratamento nas diversas fases da doença, percebe-se que, quanto mais precocemente (após o diagnóstico) se inicia o uso do imatinibe, melhores serão os resultados obtidos. Assim sendo, os pacientes em fase crônica que iniciam o tratamento mais precocemente obtêm melhores resultados do que os pacientes que vêm a utilizar a droga em fase crônica tardia ou nas fases avançadas.

Estes resultados sugerem que a maior eficácia do medicamento é na fase inicial da LMC e que o imatinibe seria útil como primeira linha na LMC. O uso mais tardio e em fases mais avançadas está associado à maior frequência de resistência à medicação.

A boa tolerância ao tratamento e as respostas satisfatórias, por outro lado, não autorizam a suspensão temporária do medicamento, uma vez que se encontra bem esclarecido na literatura o retorno da patologia após a interrupção da medicação.

Concluímos que o uso de mesilato de imatinibe em pacientes com LMC demonstra uma maior eficácia quando utilizado mais precocemente, visto que $78 \%$ dos pacientes em fase crônica, $17 \%$ em fase acelerada e $9 \%$ em crise blástica apresentaram resposta hematológica completa; e $44 \%$ dos pacientes em fase crônica e nenhum nas fases avançadas obtiveram resposta citogenética completa. Com relação aos efeitos colaterais, o trabalho mostra que a idade, a fase da doença e o tempo de uso não tiveram relação com o número de efeitos colaterais apresentados pelos pacientes. $\mathrm{O}$ mesilato de imatinibe ainda não é a solução definitiva para o tratamento da LMC em virtude dos mecanismos de resistência desenvolvidos pelo clone maligno, mas iniciou uma nova era de novos fármacos indicados para atingir alvos moleculares definidos, estabelecendo assim um novo paradigma para o desenvolvimento futuro de outros compostos farmacêuticos. A partir do imatinibe, fármacos inibidores de BCR-ABL tirosina-quinase, como o dasatinibe e o nilotinibe, foram aprovados pelo FDA para o tratamento da LMC resistentes ao imatinibe, além de estarem novos fármacos em pesquisa.

\section{Abstract}

Chronic myeloid leukemia (CML) is a genetic disorder of unknown etiology characterized by increased and unregulated growth of myeloid precursor cells in the bone marrow. CML is associated with a characteristic chromosomal translocation known as the Philadelphia chromosome. This is a descriptive observational study of CML patients in the Walter Cantidio University Hospital, Federal University of Ceará, Brazil. The aim of the study was to investigate the efficacy and common side effects of imatinib mesylate therapy. Twenty-six patients were included in the study: 9 in the chronic phase (34.61\%), 6 in the accelerated phase (23.08\%) and 11 in blast crises $(42.31 \%)$. The cases in the chronic phase had previous intolerance to interferon alpha (IFN- $\alpha)$. Complete hematological responses were observed in 7 patients: 5 in the chronic phase, 1 in the accelerated phase and 1 in blast crisis. During the first year of treatment, 4 patients in the chronic phase presented complete cytogenetic responses. One of these patients subsequently lost response. No patient in the accelerated phase or blast crisis showed complete cytogenetic response. Complete molecular response was confirmed in 1 patient in the chronic phase. Among the 18 patients who were alive at the end of the study, only 4 patients $(22.22 \%)$ had no complaint. The most commonly reported adverse events were: edema (50\%), adynamia (33.33\%), bone and / or joint pain $(33.33 \%)$, headaches $(27.78 \%)$, cramps $(16,67 \%)$, diarrhea (16.67\%), insomnia (16.67\%), itching (16.67\%), ecchymosis $(11.11 \%)$, nauseas $(11.11 \%)$, epigastric pain $(5.55 \%)$, erythema $(5.55 \%)$, shedding of tears $(5.55 \%)$, dehydration of the skin and lips $(5.55 \%)$, rush $(5.55 \%)$, and sweating $(5.55 \%)$. A minority of patients evolved with imatinib resistance. Newer drugs and trials are being developed to overcome resistance and to increase responsiveness to tyrosine-kinase inhibitors. Rev. Bras. Hematol. Hemoter. 2009;31(3):137-142.

Key words: Chronic myeloid leukemia; imatinib mesylate; pharmacotherapeutic follow-up.

\section{Referências Bibliográficas}

1. Deininger MW, et al. Allografting after imatinib therapy no evidence for increased transplant - Related mortality and favorable results in patients transplanted in remission. A Retrospective Study by the EBMT. Program and Abstracts of the $44^{\text {th }}$ Annual Meeting of the American Society of Hematology. December 6-10, 2002. Philadelphia, Pensylvania, Abstract, 3097.

2. Druker BJ, Talpaz M, Resta D J. Efficacy and safety of a especific inhibitor of the BCR-ABL tyrosine kinase in chronic myeloid keukemia. N Engl J Med. 2001;344(14):1031-7.

3. Druker BJ, Tamura S, Buchdunger E. Effects of selective inhibitor of the Abl tyrosine kinase on the growth of Bcr-Abl positive cells. Nat Med. 1996;2(5):561-6.

4. Fardel S, Talpaz M, Estrov Z. Chronic myelogenous leukemia: biology and therapy. Ann Intern Med. 1999;131(3):207-19.

5. Gambacort-Passerini $C$, Le Coutre $P$, Mologni L. Inhibition of the ABL kinase activity blocks the proliferation of BCR-ABL + leukemic cells and induces apoptosis. Blood Cells Mol Dis. 1997; 23(3):380-94.

6. Hamada G. Epidemiologia. In: Brentani MM, Coelho FRG, Iyeyashu H, Kowalski LP. As Bases da Oncologia. São Paulo: Lemar, 1998.

7. Horowitz MM, Rowlings PA, Passweg JR. Allogeneic bone marrow transplantation for CML: a report from the International Bone Marrow Transplant Registry. 1996;17 Suppl 3:S5-6.

8. Usui N. Imatinib therapy for patients with chronic myelogenous leukemia. Gan To Kagaku Ryoho. 2005;32(3):297-303.Review.

9. Baccarani $\mathrm{M}$, et al. Evolving concepts in the management of chronic myeloid leukemia: recommendations from an expert panel on behalf of the European Leukemia. Blood. 2006;108(6):1809-20

10. Kantarjian HM, et al. Imatinib (Gleevec () ) Results in sustained hematologic and cytogenetic responses among chronic - phase chronic myeloid leukemia failing interferon alfa; up to 31 months follow-up of 454 patients on phase II Study. Program and Abstracts of the 44th Annual Meeting of the American Society of Hematology. December 6-10, 2002. Philadelphia, Pensylvania, Abstract, 347.

11. Sawyers CL, Hochhaus A, Feldman E, Goldman JM, Miller CB, Ottmann OG, et al. Imatinib induces hematologic and cytogenetic 
responses in patients with chronic myeloid leukemia in myeloid blast crisis; results of a phase II study. Blood. 2002;99:3530-9.

12. Kantarjian H, Pasquini R, Hamerschlak N, Rousselot P, Holowiecki $\mathrm{J}$, Jootar $\mathrm{S}$, et al. Dasatinib or high-dose imatinib for chronic-phase chronic myeloid leukemia after failure of first-line imatinib: a randomized phase 2 trial. Blood. 2007;109(12):5143-50.

13. O'Dwyer ME, Mauro MJ, Blasdel C, Farnsworth M, Kurilik G, Hsieh YC, et al. Clonal evolution and lack of cytogenetic response are adverse prognostic factors for hematologic relapse of chronic phase CML patients treated with imatinib mesylate. Blood. 2004;103(2):451-55.

14. Tsao AS, Kantarijian H, Talpaz M. STI-571 in chronic myelogenous leukaemia. Br J Haematology. 2002;119(1):15-24.

Avaliação: Editor e dois revisores externos

Conflitos de interesse: sem conflitos de interesse

Recebido: 15/07/2008

Aceito após modificações: 15/12/2008 